\title{
Modelling the Vertical Performance of Down - Dip Water and Gas Injection (WAG) in a North Sea Sandstone Reservoir
}

\author{
Harstad O., Jakobsen K. A.
}

Statoil a. s., Norway

\begin{abstract}
Copyright 1995. Steering Committee of the European IOR - Symposium.
This paper was presented at the 8th. European IOR - Symposium in Vienna, Austria, May 16 - 17, 1996

This paper was selected for presentation by the Steering Committee, following review of information contained in an abstract submitted by the author(s). The paper, as presented has not been reviewed by the Steering Committee.
\end{abstract}

\begin{abstract}
History matching by use of a geologically charged simulation model has been performed in order to anaiyse the implementation of a proposed down-dip WAG scheme in a North Sea sandstone reservoir. The reservoir is heterogeneous consisting of fluvially deposited sand- and mudstone, and has been flooded partly by pressure support from gas injection, and partly by pressure support from an underlying water aquifer. Reservoir simulator grids with different vertical resolutions have been used for history matching.
\end{abstract}

The reservoir simulation models are used for simulation of the down-dip WAG injection flood. Heterogeneities and vertical barriers in this particular reservoir are predicted to give complex water and gas front movements.

Discussions of the representation of the heterogeneity pattern in the reservoir simulation model are given. Unpredictable water and gas front movements are assumed to make reservoir surveillance difficult. In this case, a continuous improvement of reservoir description is recommended. Use of refined reservoir models utilizing information from an intensive data acquisition program may be the key to success.

\section{INTRODUCTION}

The objective of this work was to construct a numerical reservoir model for detailed studies of the complex water and gas front movements in heterogeneous reservoir sands. The heterogeneities represented in a reservoir simulator (ECLIPSE) ${ }^{l}$ are modelled by a stochastic approach. By use of stochastic methods it is possible to construct more realistic heterogeneity patterns compared to conventional interpolation techniques. Several possible distributions of facies and petrophysical-variables can-be'produced:-

Model grids with different vertical resolutions have been tested. This paper presents a comparison between measured (based on a production $\log$ ) and simulated vertical rate distributions. Simulated and measured production well behaviour before and after recompletions are also presented.

The reservoir models are further used for predicting a downdip water and gas injection.

\section{STATIC MODELLING}

Recently, stochastic modelling techniques have proven to be an efficient way of describin! reservoir heterogeneities in 3 dimensions. Stochasti inodelling has been a tool for better transfer of information between disciplines. Geoscience personell, from petrophysicists, geologists, sedimentologists. and reservoir engineers perform their work together with this tool. This results in easier estimation of reservoir parameters (including uncertainty ranges), as it provides visualization of the interaction and influence of the different reservoir parameters.

Modelling of heterogeneities in this particular study is divided into four stages:

- Generating 2-D surfaces to restrict the modelling area.

- Generating distribution of facies bodies within the volumes bounded by these surfaces. 
- Generating of petrophysical properties within each facies body.

- Performing upscaling to the scale used in the dynamic reservoir simulation model.

The stochastic modelling tool used by Statoil is Storm ${ }^{2}$. Storm is able to connect all this stages in an efficient way. so that we are able to maintain the consistency when transfering data between the different stages.

\section{Surfaces}

The zonation of the reservoir is based on the results of a high resolution stratigraphy study. The limits of the zones are defined by the flooding surfaces which are fairly smooth. Surfaces are generated by kriging. All true vertical thicknesses observed in the wells are used as conditioned data points.

More complex surfaces; sequence boundaries, occuring between the flooding surfaces, are modelled in an implicit way, using a vertical trend of channel belt distribution within each zone.

\section{Facies}

A combination of fibre processes and Gaussian Random fields ${ }^{3}$ was used to produce the channel systems required to model the depositional environment. The basis of this method is to introduce lines which represent the channel belt direction. Then for each line the number of riverbeds is drawn from a given distribution. The individual river-beds within these channel belts are modelled using correlated one dimensional Gaussian random functions to describe their relative location, thickness, and width. Well observations are taken into account to condition simulations to observed data.

The three uppermost zones shows repeating sequences of high-stand and low-stand systems (see Fig. 1 and Fig. 2 ). The repeating sequences are modelled in three zones, each consisting of one low-stand system at the top and a high-stand system at the bottom of the zone. Since highstand and low-stand systems are significantly different regarding geometries of bodies and net/gross ratios, the two systems are modelled independently. Afterwards the two realizations are merged together. Modelled facies types are stacked low-stand channels, single-story highstand channels, crevasses connected to the high-stand channels, and overbank facies.

The input parameters required for the modelling are based on available field data, analogues, and general geological knowledge.

The incised valleys in the low-stand systems are filled by combining several channel belts. In the low-stand system most of the effort was put into establishing parameters for the geometry of an incised valley. In the high-stand system the work was concentrated on modelling the single channel elements. Geometry parameters for each channel were established. In addition, parameters for crevasses relatively to the channels were established. The overbank facies are penetrated by the other facies types. By modelling this way, the repeating sequences of high-stand systems and low-stands ystems are reproduced in a sensible way.

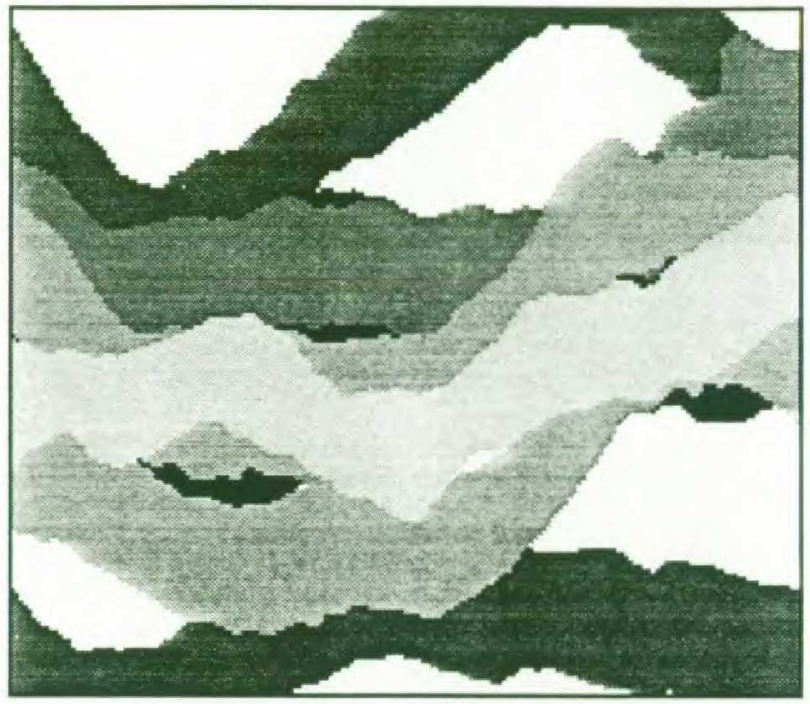

Fig. 1. An example of Low-stand Channels in modelling zone number 1

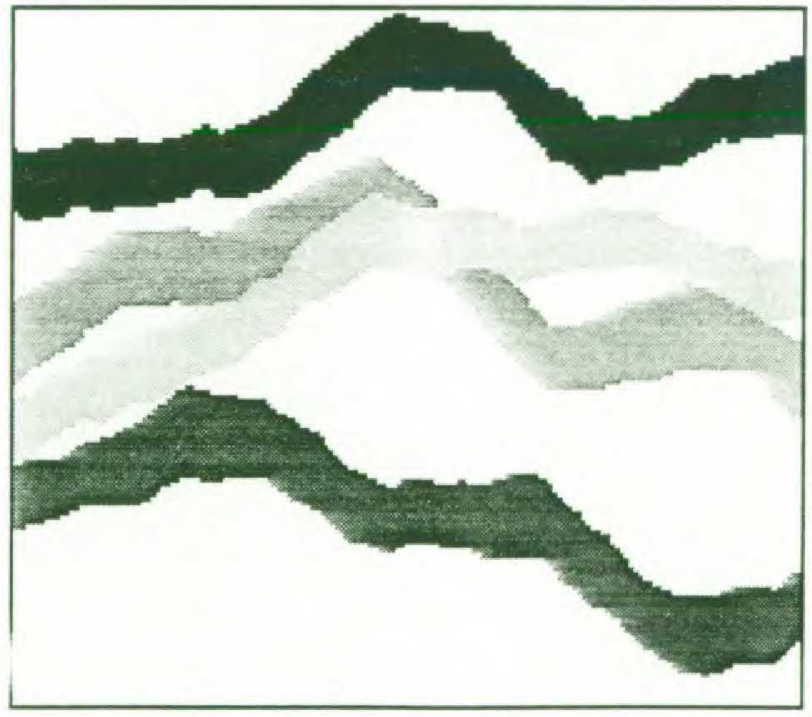

Fig. 2. An example of High-stand Channels in modelling zone number 1

In the lowermost zone another approach was employed. It was impossible to correlate the different incised valleys. Thus only one type of channel facies was used for the whole zone. In addition to the channels, crevasses, overbanks, and sheet floods were also modelled in the zone. 


\section{Petrophysical parameters}

Mapping petrophysical parameters within a given facies was the next step to be performed in the modelling work. The facies model is used as input to the petrophysical modelling, assuming that each facies has its own petrophysical property distribution and that they can be modelled as correlated Gaussian Fields. For each of the observed facies, permeability, porosity and water saturation distribution were modelled. This step was performed on a $3 \mathrm{D}$ regular grid with a cell size of approximately $100 \times 100 \times 0.5$ meters.

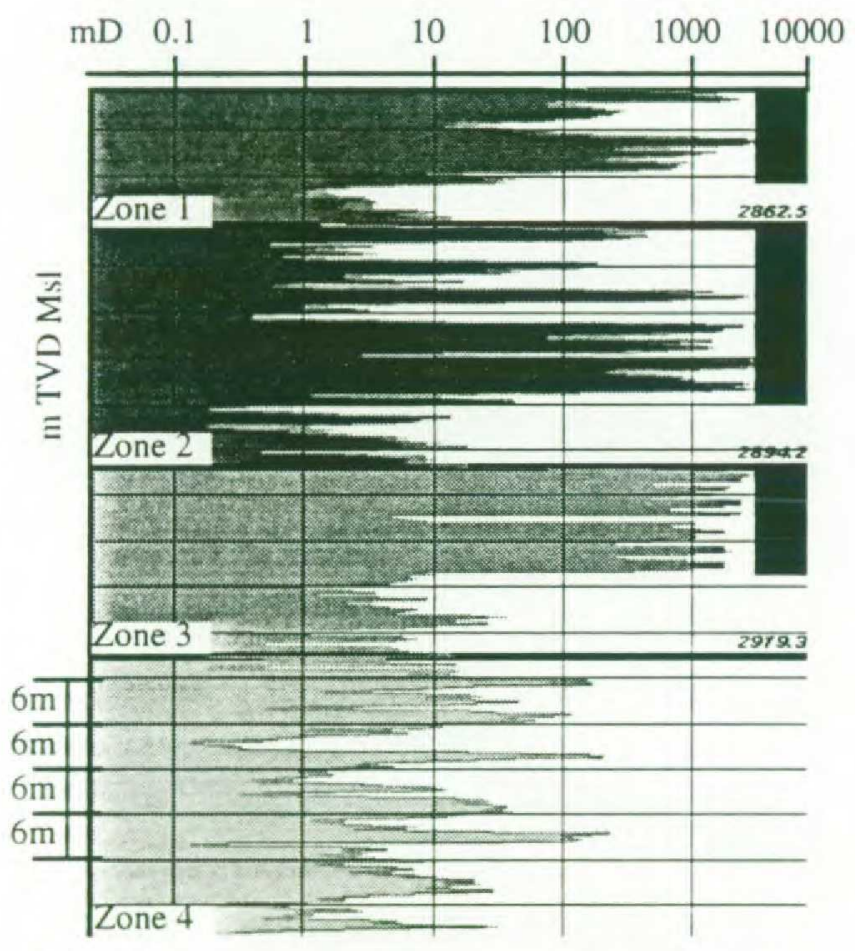

Fig. 3. Example of a permeability log for all four zones. The black bars to the right illustrates the Low-stand systems with good reservoir quality. As seen, zone 4 is not typical.

\section{DYNAMIC MODEL}

\section{Upscaling}

Reservoir simulators cannot handle as many grid blocks as used in the static modelling of reservoir blocks. Consequently, it is necessary to reduce the number of grid cells in a suitable way. Porosity and water saturation are upscaled by arithmetical averaging within a reservoir simulator grid block (water saturation is weighted by porosity). The upscaling of absolute permeability is performed by solving Darcy's flow equation with mass conservation three times to caiculate the permeability in $x, y, z$ - direction for the reservoir simulator grid. By use of element models with high vertical resolution, it is required most effort to upscale the vertical permeability.
There are four modelling zones in this element model:

- Zone 1: 40 grid layers

- Zone 2: 28 grid layers

- Zone 3: 40 grid layers

- Zone 4: 65 grid layers

The number of layers in the four fine-scale zones adds up to 173 layers in the stochastic model. Reservoir simulator grids (ECLIPSE) ${ }^{1}$ containing 12 layers, 23 layers and 45 layers are generated. These models will be further used for predicting dynamic behaviour.

The $\mathrm{X}$ - and Y-length in the modelling grid are both approximetely 100 meters. Compared to the grid of ECLIPSE with corresponding values of $100 \times 220$ meters, the difference is not significant (see Fig. 4). The large scale flow elements, the fluvial channels, are still wider than an ECLIPSE grid block. That means upscaling in $\mathrm{X}$ - and $\mathrm{Y}$ - direction is not expected to introduce a major error.

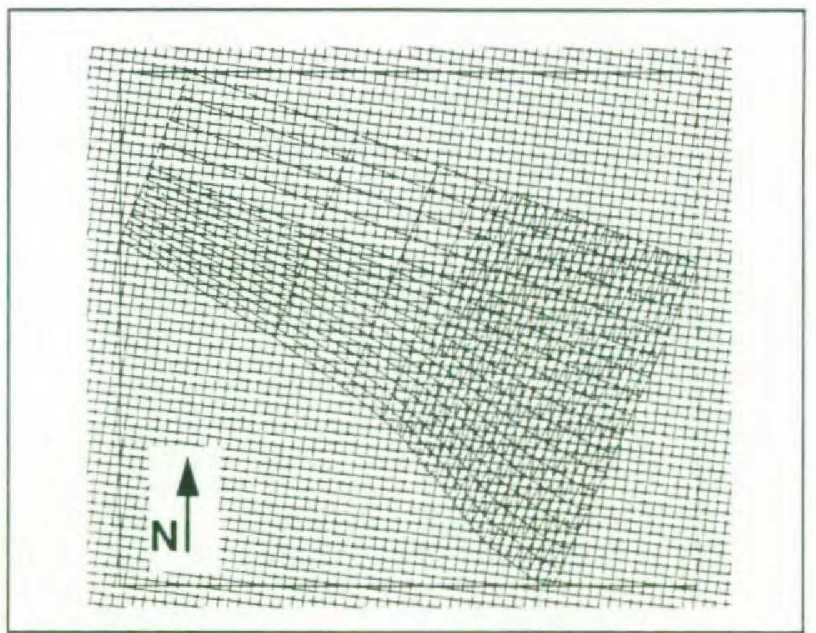

Fig. 4. Overview of fine scale modelling grid compared to Eclipse grid

\section{Fluid modelling}

The reservoir fluid in this field shows minor variation in physical properties and molecular composition with depth and area. Several tests indicate a minimum miscibility pressure in the range of 320 to 350 bars. depending on the molecular composition of the injected gas. In this study miscible conditions are assumed throughout the simulation period.

The miscibility is modelled using a combination of the Todd-Longstaff ${ }^{4}$ and Lanz ${ }^{5}$ methods, taking into account effective viscosity changes corresponding to a ToddLongstaff omega parameter of 0.25 . 


\section{Reservoir Parameters}

Porosity, permeability and initial water saturation are provided from the stochastic model. Residual oil saturation after water-flooding is calculated as a function of initial water saturation, corresponding to approximately $15 \%$ in average.

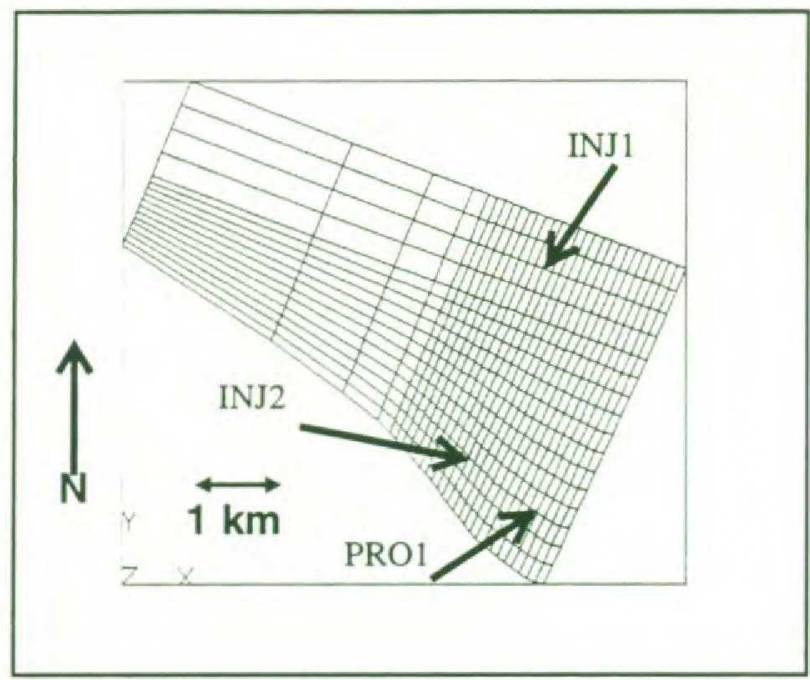

Fig. 5. Overview of Eclipse grid with well locations.

\section{HISTORY MATCHING}

\section{Reservoir Status}

The reservoir has been flooded partly by pressure support from gas injection, and partly by pressure support from an underlying water aquifer. The produced oil volumes constitute about $20 \%$ of original oil in place.

The production from the reservoir will take place from drainage points located structurally high. The injected gas has vapourized attic oil, and Compensated Neutron Logs (CNL) have indicated that a gas cap is established at the top of the reservoir.

The original reservoir pressure was 404 bars, referred to a datum depth of 2700 mTVDSS. The pressure drop in the reservoir is approximately 50 bars.

There is one production well (PRO1) and one injection well (INJ1) with historical performance within the simulated element (see Fig. 5).

\section{Completions and Production Logging (PLT)}

The oil producer (PRO1) was completed and initially perforated in November 1985. Five extra perforation intervals were added in the lower part of the reservoir in March 1990 (see Fig. 6).

The injector (INJ1) was perforated in the 4 uppermost zones. The well began injecting gas in November 1981.

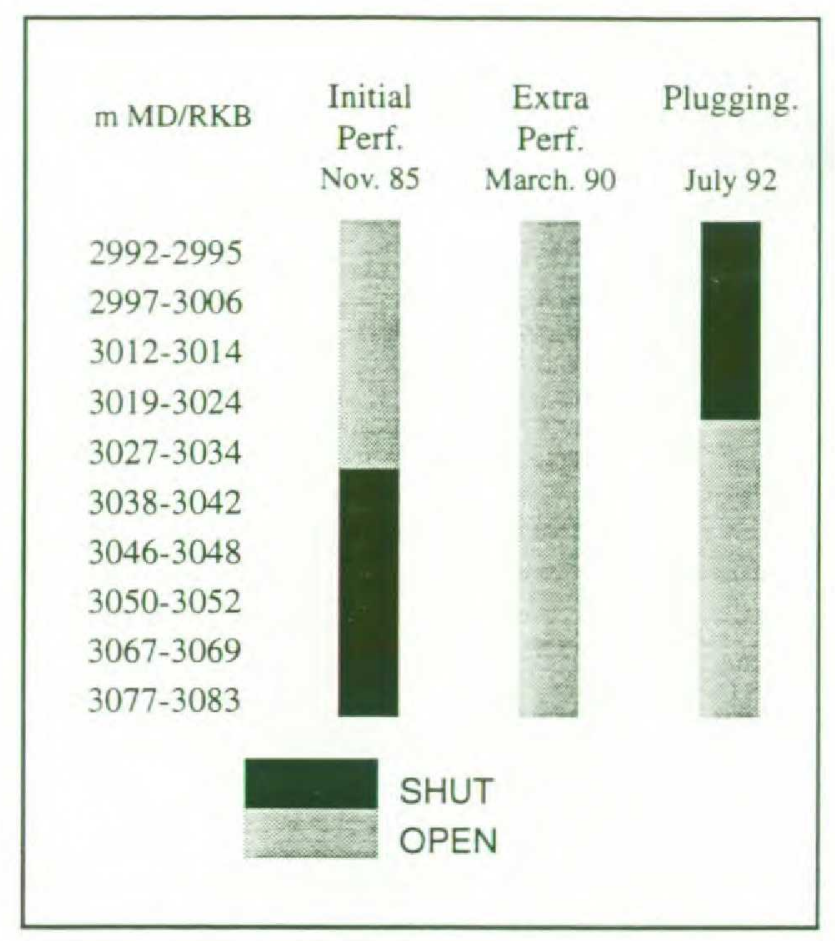

Fig. 6. Perforations/Reperforations

Three production logs (PLT) have been run in well PRO1. The purpose of these logs was to determine which layers were actually producing oil, water, and free gas. The PLT logs show that the main contribution of free gas came from the two uppermost perforation intervals, and the water was produced from the lowermost perforation interval. There was no indication of flow behind the casing, and no significant cross-flow was registered during shut-in.

The recommendation, based on a PLT log run in April 1992, was to plug the upper perforations in order to reduce the gas production. Hence, the 4 uppermost perforation intervals were plugged in July 1992 (see Fig. 6).

\section{History Match of the Vertical Flow Distribu- tion}

Three dynamic element models, as described earlier, containing 12, 23 and 45 layers, are used for reservoir simulation and comparisons with historical data from the production well (PRO1). The objective of this investigation is to optimize the number of model layers, and yet adequately depict the reservoir heterogeneities in a proper way.

Monthly rates of production (PRO1) and injection (INJ1) are input parameters in the element models. Some flux occurs across the element borders. In order to match the exact offtake in the element, a dummy well is introduced at the northern border to account for flux out of the element. The outflux rate is based on results from full field simulations. 
The measured oil rate from the producer is reproduced in the simulation models. Reservoir pressure is satisfactorily matched for all models. There is, however, a considerable difference in gas and water production in the models. A comparison between simulated and measured rates before and after recompletions of the production well (PRO1), show that refinement of the model grid improves the history match.

Following the opening of perforations further down in the reservoir in March 1990, the well began producing water from the lowermost perforation in September 1990 (see Fig. 7). Simulations by use of the 45 layer model show a rather good match. The 12 layer and 23 layer models predict no formation water production before January 1991. The increase in water cut is also slower compared to measured data (see Fig. 7). The simulation results indicate that a refinement of high permeable channels results in an earlier water breakthrough and faster build-up.

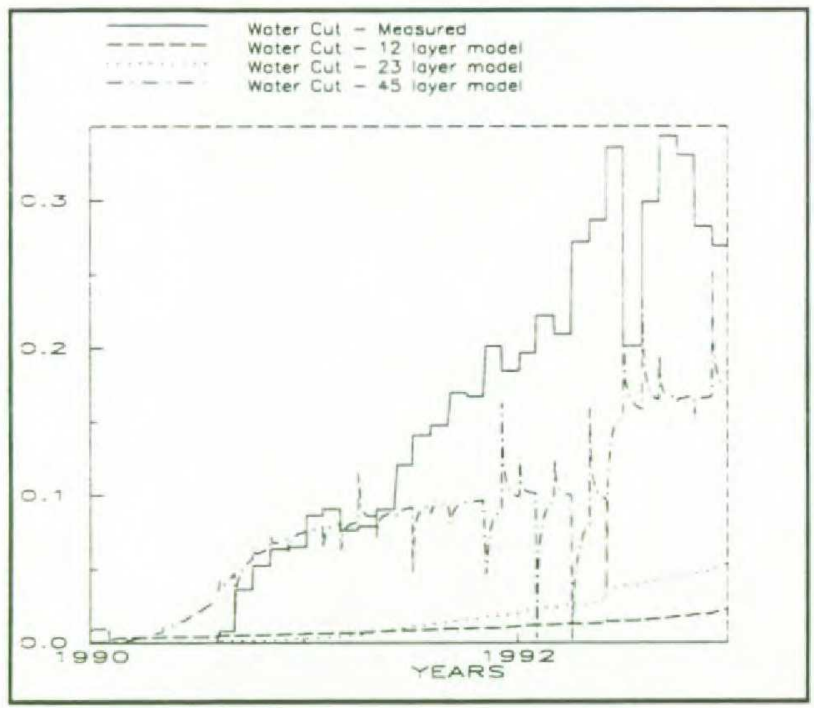

Fig. 7. Water Cut in well PRO1 before and after recompletion in March 1990

The well PRO1 has produced free gas since August 1987. The plugging of the 4 uppermost perforations in July 1992 results in a temporary decrease in GOR (see Fig. 8).

The temporary decrease in free gas production is achieved in the 45 layer model. Because of grid refinements inside channel belts, gravity dominated gas tonguing further down in the reservoir is satisfactorily represented.

In the coarser models, with model grid containing 12 and 23 layers, the gas migrates to the top of the reservoir, due of gravity segregation. Therefore, no free gas was predicted from well PRO1 after plugging of the uppermost zones (see Fig. 8).

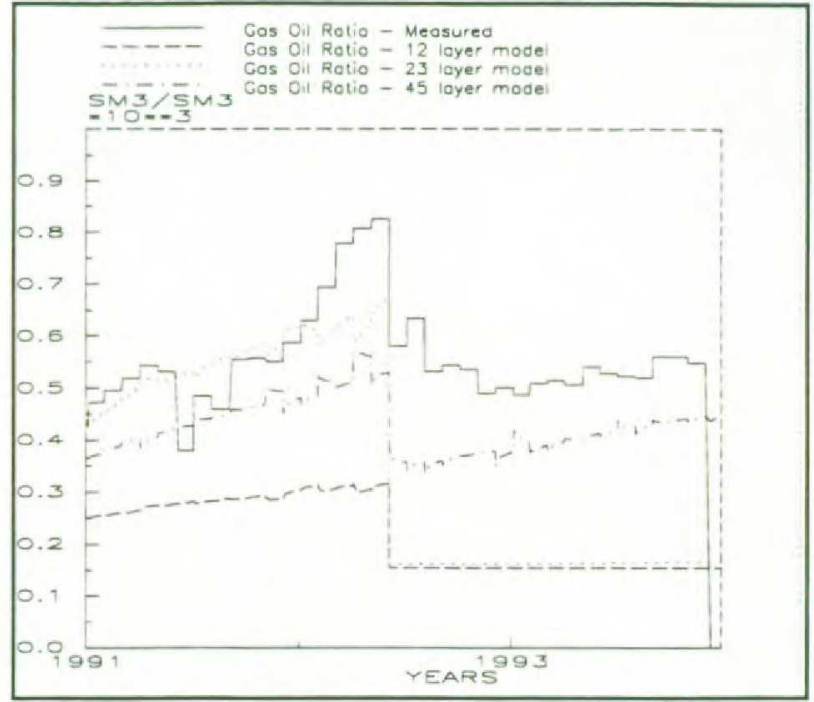

Fig. 8. Gas Oil Ratio in well PRO1 before and after plugging of the 4 uppermost perforations in July 1992

A comparison between simulated vertical rate distributions and a PLT log illustrates that a refinement of 45 model layers is needed to model satisfactorily the vertical flow performance (see Fig. 9 and Fig. 10).

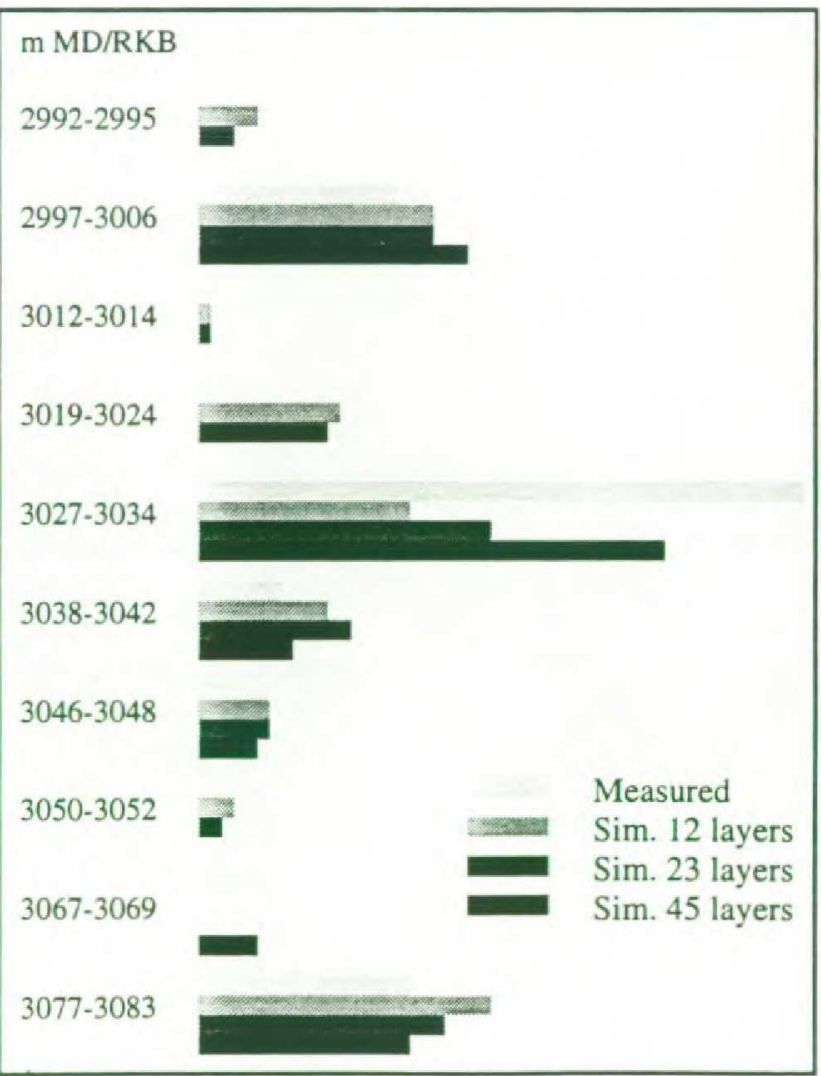

Fig. 9. Vertical Distribution of Oil Rate - PLT Log in April 1992 


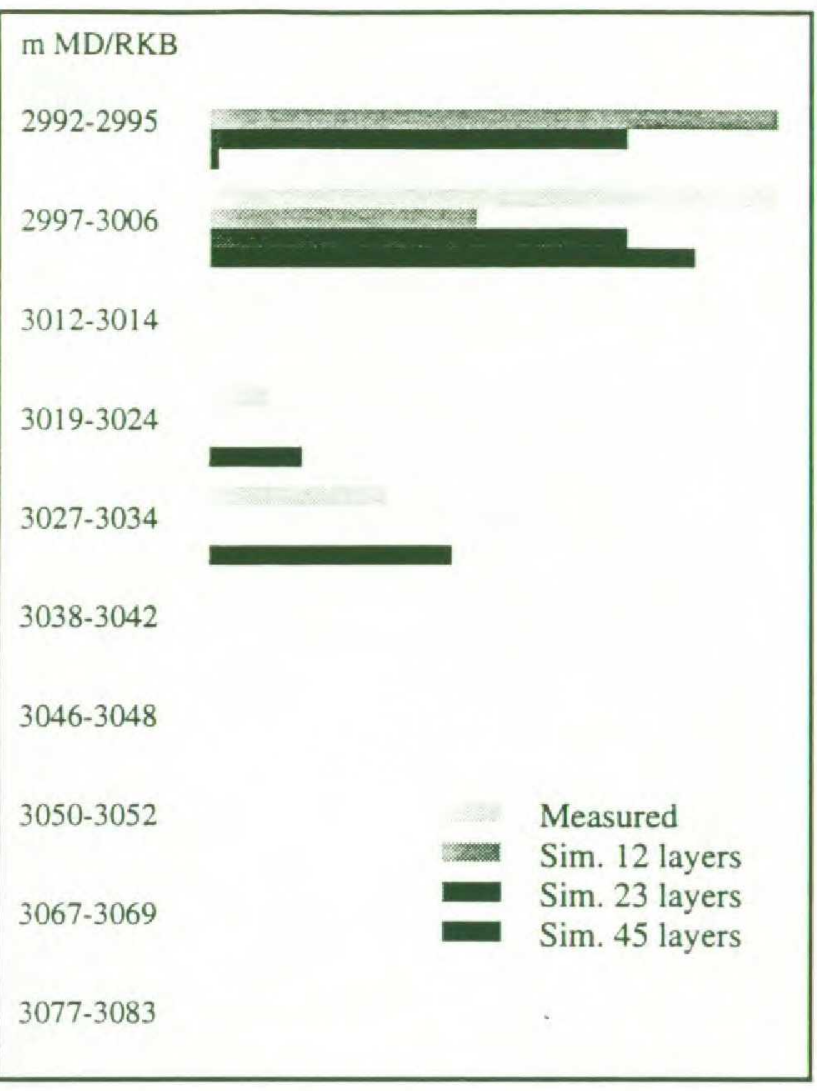

Fig. 10. Vertical Distribution of Free Gas - PLT Log in April 1992

\section{WAG-MODELLING}

\section{Model input}

The proposed future flooding strategy for the reservoir is a down-dip miscible water alternating gas injection flood for pressure maintenance and effective volumetric sweep. It is preferred to maintain reservoir pressure above $330-340$ bars to ensure miscible conditions in the reservoir.

The well INJ2 (see Fig. 5) was simulated to start injecting water from March 1996. The injector is perforated in all reservoir zones. The water injection continues until start-up of gas injection in January 1997. From 1997 until the end of the predicted period, gas is injected alternating with water with cycles of 0.5 year. The injection of water and gas is to balance the offtake from the element through voidage replacement.

\section{Simulation Results}

The starting-point for WAG predictions is different in the three reservoir models (12, 23 and 45 layers). As described earlier, the 45 layer model reproduces the production history satisfactorily. The model has predicted a higher GOR in the producer and a wider areal extension of the gas front compared to the coarser models. The profiles give, however, useful information concerning the flooding mechanism and grid refinements in modelling the flood strategy.

A comparison of simulated water cut for the three reservoir simulation models (see Fig. 11) indicate that higher grid resolution and thus smaller scale of heterogeneities, give slower increase in water cut. These wider areal extensive and thinner water fronts, due to greater vertical resolution within the channels, give a less dominant water breakthrough.

The GOR profiles (see Fig. 12) indicate that high vertical resolution gives an earlier gas breakthrough because of improved vertical resolution within the channels. Moreover, increasing the vertical resolution preserves the transmissibility contrasts between channels belts and shales.

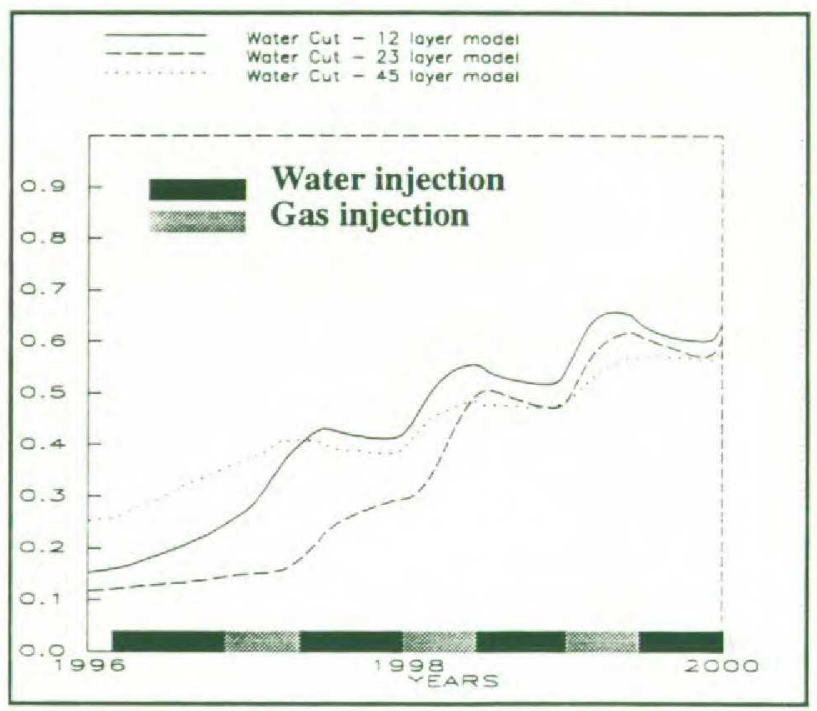

Fig. 11. Simulated Water Cut in Well PRO1

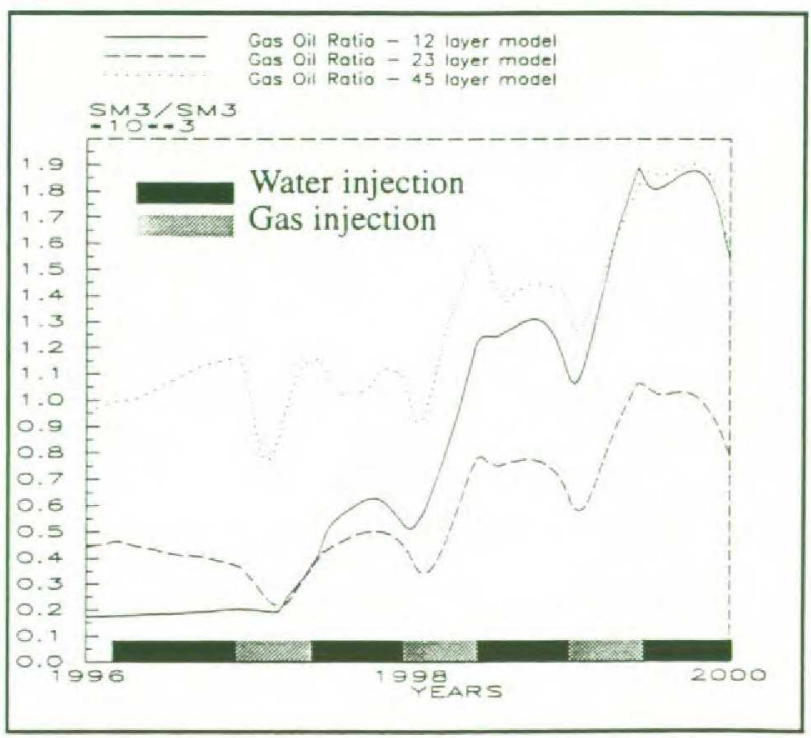

Fig. 12. Simulated Gas Oil Ratio (GOR) in Well PRO1 


\section{Flow mechanisms}

Traditionally in the North Sea, flow during WAG injection consists of two separate fronts; a gas/oil and an oil/water front. Most frequently the injected gas migrates rapidly to the top of the formation and accumulates in a gas cap ${ }^{6}$. The front is in general governed by gravity. The velocity of the gas/oil front is higher, because of high gas mobility. The water fills parts of the reservoir below the gas/oil front. The residual oil saturation after water flooding is usually higher than for gas flooding. Therefore, the water flooded zone should be reduced to recover more oil.

The simulation results from downdip WAG in this particular reservoir, show that vertical barriers in the reservoir cause a flow pattern of water and gas which follow along the channel belts. Based on results from history matching of vertical flow given in this paper, it is anticipated that the 45 layer reservoir model represents the reservoir heterogeneity satisfactorily (see Fig. 13 and Fig. 14).

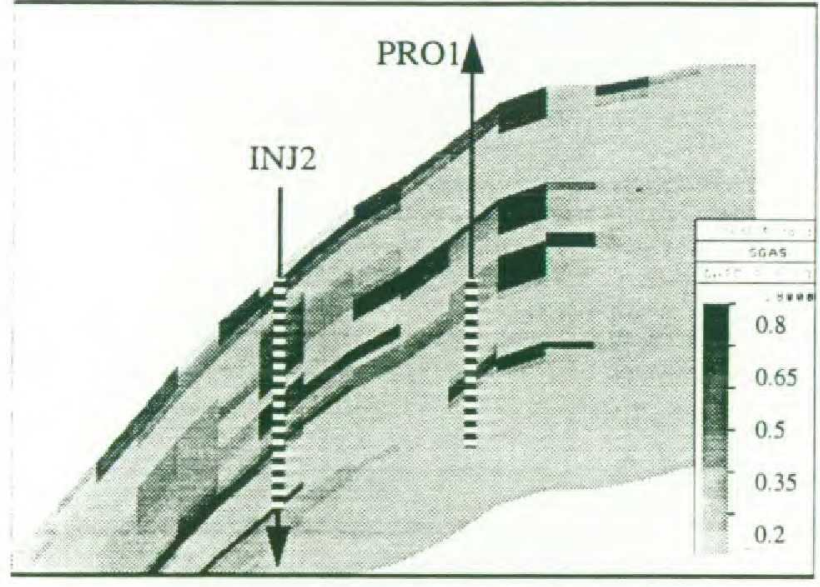

Fig. 13. Cross section of $\mathbf{4 5}$ layer model - Simulated Gas Saturation July 1998.

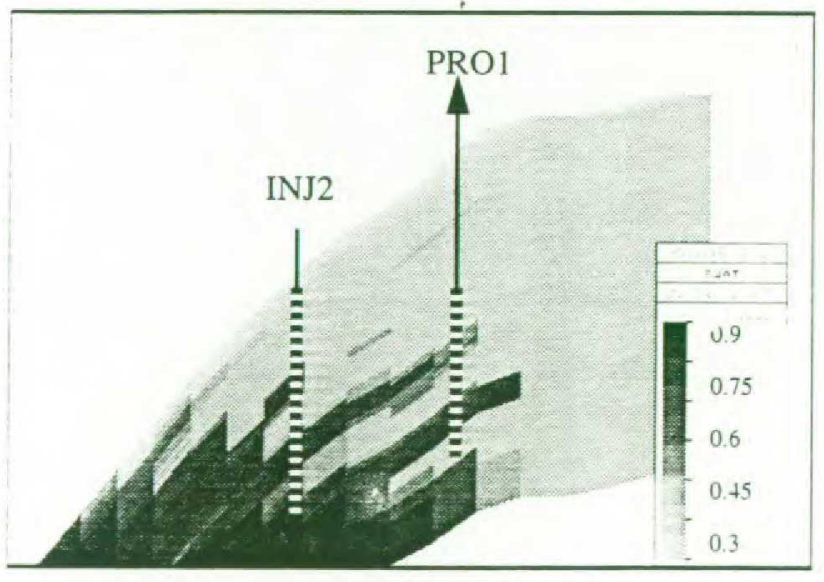

Fig. 14. Cross section of $\mathbf{4 5}$ layer model - Simulated Water Saturation January 1998.
Using a coarser model (12 layers) the vertical migration profile within the sand bodies is not preserved. In this model a sand body is represented by only one model layer, and consequently there are no transmissibility contrasts between channels belts and shales (see Fig. 15 and Fig. 16).

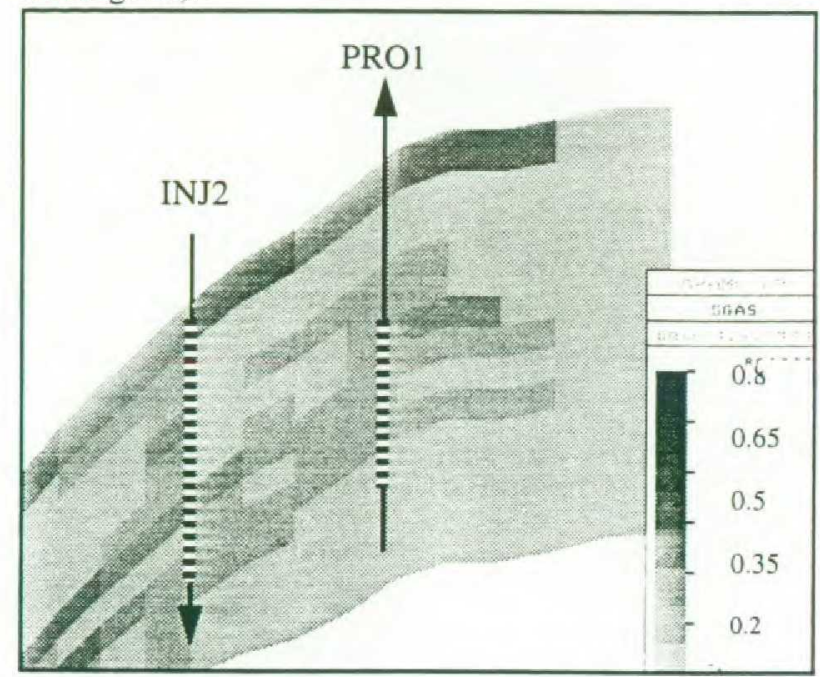

Fig. 15. Cross section of 12 layer model - Simulated Gas Saturation July 1998.

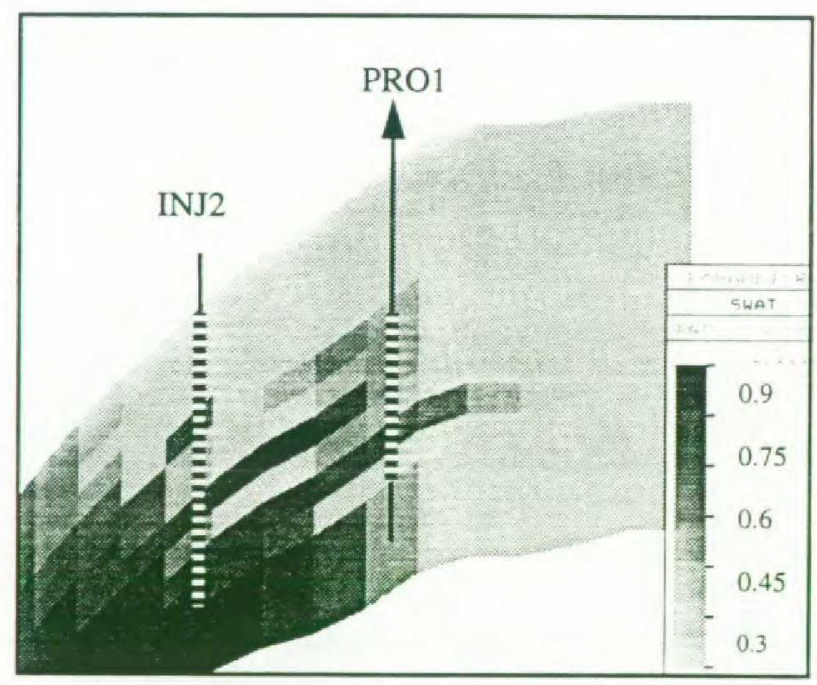

Fig. 16. Cross section of 12 layer model - Simulated Water Saturation January 1998.

The overall sweep efficiency of the presented reservoir is expected to be relatively poor, because of the heterogeneous nature of the fluvially deposited formation, In the downdip water and gas injection flooding process, reservoir heterogeneities seem to have a significant effect on both the areal and vertical sweep efficiency. In addition, gravity tonguing of the gas over the oil bank makes the flow pattern complicated. 
The main uncertainty of the downdip WAG simulations is how rapid the gas front may advance through the reservoir. This is influenced by high permeable streaks, internal faulting and reservoir heterogeneities. Using models with high vertical resolution, the process effectiveness will, to a lesser degree, be a function of residual oil saturation. The gas is expected to rapidly migrate to the top of each sand body and follow along the channels belts. The thicknesses of each channel belt that is flushed by gas will significantly influence the effect of the downdip WAG injection efficiency.

\section{Reservoir Monitoring}

The proposed down-dip WAG is assumed to result in complex water and gas front movements that makes the reservoir surveillance difficult. A successful monitoring of the water and gas fronts will be useful to ensure an optimal flooding of the reservoir. Back-up options must be evaluated with the aim to make corrective actions as early as possible. To achieve this goal it is important to establish a reservoir model utilizing information from an intensive data acquisition program. Updated reservoir information, based on the data acquired ought to be implemented in the future reservoir simulation model to establish more reliable WAG performance prognosis. Use of refined element models is supposed to improve the understanding of the dynamic changes in the reservoir during WAG flow implementation.

Different stochastic realizations and different grid refinements in the reservoir models can be constructed in an efficient way by use of Storm ${ }^{2}$. Grid refinements and realizations are recommended for future history matching of the WAG flood.

\section{CONCLUSIONS}

The following conclusions are drawn from the study:

1. The detailed geological model, including high vertical resolution, has enabled us to perform detailed predictions of the WAG strategy for this heterogeneous reservoir.

2. The stochastic modelling tool used is able to connect all stages for reservoir model constructions in an efficient way while maintaining consistency when transfering data.

3. Increase of the vertical resolution in the reservoir model preserves the transmissibility contrasts between channel belts and shales much better, and improves the history match of vertical flow performance significantly.

4. Use of different stochastic realizations and vertical grid refinements of the reservoir model are recommended for history matching and reservoir monitoring of the WAG scheme.

\section{ACKNOWLEDGEMENT}

The authors wish to thank Statoil for the permission to publish this paper.

\section{NOMENCLATURE}

$\begin{array}{ll}\text { mTVD Msl }= & \begin{array}{l}\text { True Vertical Depth refer to mean } \\ \text { sea level. }\end{array} \\ \text { mTVD ss }= & \begin{array}{l}\text { True Vertical Depth refer to sub sea } \\ \text { level. }\end{array} \\ \text { mMD RKB }= & \begin{array}{l}\text { Measured depth refer to rotary } \\ \text { Kelly Bushing }\end{array}\end{array}$

\section{REFERENCES}

1. "ECLIPSE 100, Reference Manual, Version 94A", Intera Information Technologies Limited. Abingdon, England (1994)

2. Bratvold, R. B., Holden, L., Svanes T. and Tyler, K.: 'STORM: Integrated 3D stochastic reservoir modelling tool for geologists and reservoir engineers.' Second European Petroleum Computer Conference. Proceedings SPE 27563, Aberdeen, March 15-17, 1994.

3. Georgsen, F. and Omre, H.: "Combining Fibre Processes and Gaussian Random Functions for Modelling Fluvial Reservoirs", Proc.4th Geostatistical Congress, Troia, Portugal, Sept. 1992.

4. Todd, M.R. and Longstaff, W.J.: "The Development, Testing, and Application of a Numerical'Simulator for Prediction of Miscible Flood Performance," JPT (July 1972), 874-882.

5. Lanz, R.B.: "Rigorous Calculation of Miscible Displacement Using Immiscible Reservoir Simulators," SPEJ (June 1970), 192-202.

6. Dalen V. , Instefjord R. and Kristensen R. : "A WAG injection pilot in the Lower Brent formation at the Gullfaks Field", 7th European IOR Symposium in Moscow, Russia, October 27-29. 1993. 